\title{
Original Achievements and Dynamic Quality Analysis on the Roto-Translation Servo Linear Actuator for Machine-Tools and Robots
}

\author{
Radu-lacob COTEȚIU, Vasile NĂSUI, Adriana-Gabriela COTEȚIU
}

\begin{abstract}
The paper presents some of the original achievements of unit elements of practice of the telescopic linear actuator type with the possibility of application in industry. We also analyze some parameters of dynamic quality that influence their technical characteristics as a mechatronic product making them compatible with the requirements of such intelligent machines. The paper presents an analysis of roto-translation systems, that can be programmed, and the project of an equipment of translation helped by a system which changes the rotation movement into a translation movement, using a screw with balls. The main constructive variants, the model of kinematics, dynamics and the calculus with the working conditions are also presented. The result of the research can be valued immediately in any system of linear movement because it is based on the newest techniques in the field of modeling. Their objective is the developing of a new system of electro-mechanic linear actuator type, as well as perfecting the existing ones in a very short time and in highly energy-efficient and economic conditions. In the future these researches can continue to develop new applications on other types of mechanical transmissions using this method and different other modular controls. Finally, additional researches on defining the correct set of requirements and specifications for new electro-mechanic actuator systems could significantly improve their development.
\end{abstract}

Keywords: dynamic quality; electro-mechanic linear actuators; mechatronic; motion control; optimal synthesis

\section{INTRODUCTION}

The technical and technological evolution towards the integration in mechatronics included the stages of development of the module of linear acting of the actuator type marked by the integration of the microprocessors. The new generation of modern machine and installations requires power and very high speeds, precision of regulating the position and a rational coordination of the composing individual action [1].

The development of the mechatronic technology within this context contributed to the development of the actuators as elements of execution which transfer energy and information besides the cinematic chain [2, 3, 4].

The linear actuators are used for the mechanic servo systems of positioning, as systems of transmission and analogical servo systems for following the position [5].

The technical system of the present linear actuator has some characteristics which give it more efficiency in prediction and highly accurate, adjustable acting, numerical control etc. [6].
The actuator has a complex high kinematics and dynamic precision structure.

Most of the requirements on the actuator to be fulfilled are $[7,8,9]$ :

- lower energy consumption;

- the lowest specific weight without affecting the resistance and the rigidity of the whole assembly;

- secure starting and functioning within large limits of the environmental temperature;

- balanced forces and inertia couples of the weights involved in the movements;

- adopting a non uniformity degree that does not affect the smooth functioning of the machine and adaptability at the functioning regime of the machine

- secure functioning of the actuators;

- fulfilling some factors that influence the use of the actuators.

The constructive scheme of the actuator is shown in Fig. 1: planetary reducer (1), asynchronous electric engine (2) and static converter of frequency (3).

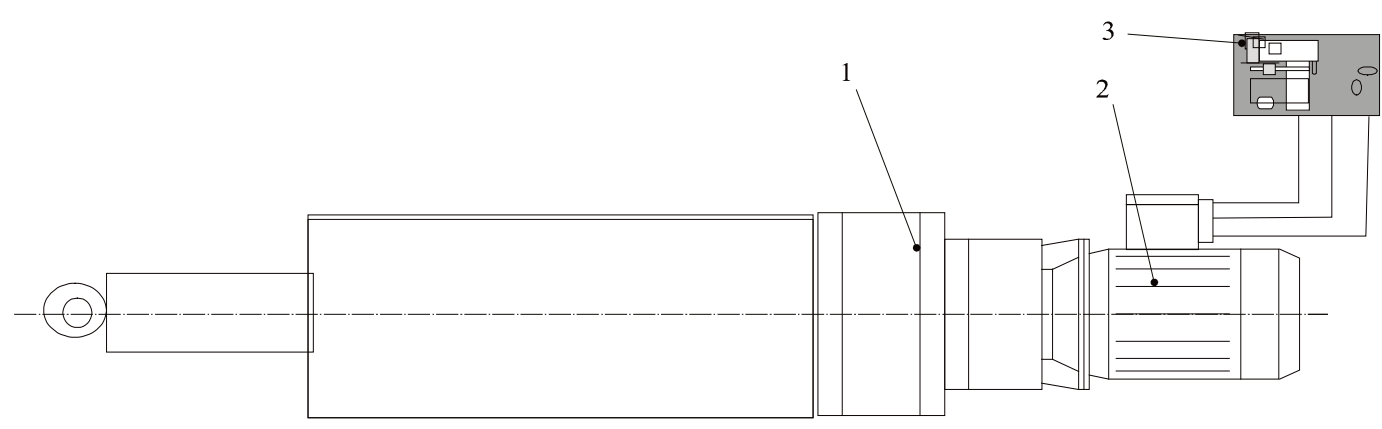

Figure 1 The linear actuators with converter: 1 - planetary reducer; 2 - asynchronous electric engine; 3 - static converter of frequency

Actuators include a lead screw, a gear train and brushless motors. Positive feedback is provided by an absolute encoder that provides absolute position feedback to the controller. Planetary gear train connects all linear actuators. Linear mechanism comprises a screw in which the screw nut can rotate and generate linear motion on the nut along the screw [10]. When a position along the screw is commanded, the motor turns the screw until the nut reaches the desired position. The thrust force is transmitted from the drive nut to the translation tube. One of the types is the electromechanical actuator, which converts the torque of an electric rotary motor into linear mechanical thrust. 
To obtain the torque of screw, we need the average force to load the system, the pitch and the efficiency of screw. The function of coordination and mechanic timing is replaced with an electronic alternative, using a new concept of dynamic regulation, which improves the performances of the system [11].

The linear actuator, as mainly electromechanical servomechanism in the industry of machine-tools and robots, has an important role in the evolution and its applications, with direct dependence on their performances. Their synthesis represents an issue which is always approached and developed in the recent literature and researches. The study of the moving control system which characterizes the dynamic answer can be simplified by the introduction of the operational function concept of transfer from the theory of the automatic systems.

The ideal dynamic behavior of a component within the control system of the movement can be defined in a single equation representing the system of null order, deducted by a differential quadratic equation written according to Lagrange-D'Alembert's principle where the elements of accumulation and dissipation of energy are missing.

$y_{e}=k \cdot x_{i}$

For the proportional automatic regulator, we have a proportionality relation between the input signal $U_{r}(t)$ and the command $U_{c}(t)$ :

$U_{c}(t)=k_{R A} \cdot U_{r}(t)$

where $k_{R A}$ is the amplifying factor of the automatic regulator.

In such system, the transfer function will be:

$$
H_{R A}=\frac{U_{C}}{U_{R}}
$$

The general solution of differential equation is obtained after solving the characteristic equation corresponding to the basis equation of the order which is homogeneous,

$$
\alpha D^{2}+\beta D+\gamma=0
$$

where $D$ is the differential generator $\mathrm{d}(d f)$.

If the equation is transcribed by introducing the differential operator $D$ and by defining in general the input signal $X_{i}$, a series of derivatives of different orders is obtained by solving a continuous equation, and the following rapport between output and input is obtained:

$$
\frac{X_{e}}{X_{i}}(D)=\frac{\lambda^{n}+\mu D^{n-1}+\gamma D^{n-2}+\ldots}{\alpha D^{2}+\beta D+\gamma}
$$

This relation indicates the operational function of transfer and the representation of the block schemes of the control systems when the dynamic answer is established.

Analysis of the preoccupations regarding synthesis of the movement control of the actuators, results in the necessity of its extension and generalization for the actuators used in the automat regulating systems. From the constructive and functional points of view, the actuators have certain kinematic and dynamic restrictions that must be considered so as not to cause significant degradations of the performances of the controlled system regarding its stability and feasibility.

The characteristics involved in the analysis of the dynamic behavior include: index of velocity, acceleration and space, frequency characteristics etc.

The control of some parameters of those transmissions like stability requirements, precision, transitory answer etc., the compromise between the accepted measure of the deviation, stationary and the desired degree of dynamic stability, is done by "correction". This is possible using a net with appropriate configuration of parallel type introduced in the reaction loop of the servo-system. The block-scheme of a servosystem of control with the transfer functions of the component elements for the electromechanical actuator with reaction loops of speed and position is presented in Fig. 2.

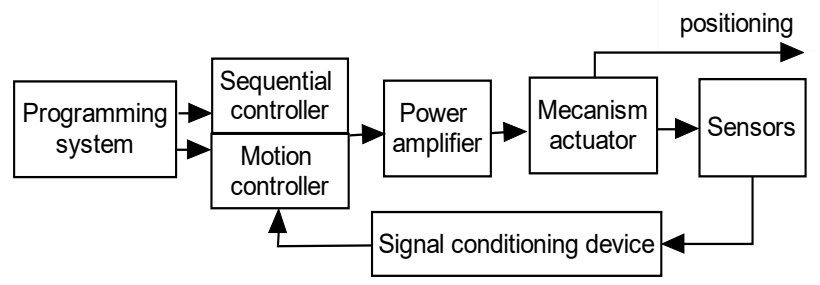

Figure 2 The block-scheme of a servo system of control with reaction loops

\section{THE OPTIMAL SYNTHESIS OF LINEAR ACTUATORS}

The optimal synthesis of the actuators of servomechanisms must fit the requirements of stability, precision, transitory avower etc. For this, a compromise between the desired degree of functional stability and the allowable size of the stationary loops is necessary. The servomechanisms used in machine tools are usually equipped with reaction loops of speed and position and their functioning is characterized by the transfer function of the engine mechanism burden system [12].

The characteristics of all elements have a special importance because they influence differently the functional parameters, resulting in the appropriate introduction of some new criteria, extra appreciations in the case of mechatronic products, presented further in Fig. 3.

Sensitivity is a measure of the dependence of the characteristics of the system on one of its elements. For the automatic systems with reaction loops we define the sensitivity of the system $S_{k}^{m}$ towards an element $k$, the global transfer function being:

$M(S)=\frac{C(S)}{R(S)}=\frac{K_{1} \cdot G(S)}{1+K_{2}(S)}$,

where: $R(S)$ is change into the entrance signal; $G(S)$ is transfer function of the direct way; $K(S)$ is transfer function of the reaction; $C(S)$ is change into getting out signal. 
According to the relations (1) and (2) it results that any change in the parameters and characteristics of the interpreters $K_{1}, K_{2}, K_{3}$ is reflected in the change of the function of global transfer of the system. From the analysis, it results that the error of the direct way (the transforming mechanism) is divided by $1+K_{2} G$, while the error owing to the reaction is added directly to the function of global transfer of the system.

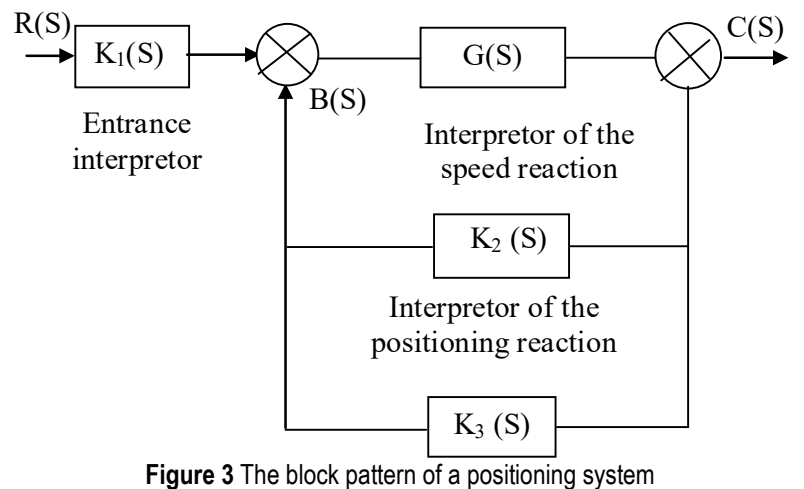

We conclude that from the point of view of the sensitivity, the error induced in the system in a direct way is less critical than the error introduced in the system from the reaction.

In the study of dynamics of the modern mechanical transmissions, the transitory phenomena are inherent, the reason why parameters as mechanical impedance become extremely important in correlation with the mechanical efficiency. Considering the influence of the mechanical impedance on energy consumption, as well as its effect on the dynamical parameters and feasibility especially in the transitory regimes, knowing its value is important [13].

At present calculation methods for efficiency, the impact of the inertia forces is neglected, as premises which satisfactorily describe reality outside the transitory regime and of the neglected friction from the rotation coupling as compared to the values of the losses within the gearing [14]. The increase of the calculation precision for the best choice of driving is done by correctly establishing the dynamic inertial couple by the evaluation of losses due to friction which leaves the angular speed transmission law unchanged, but modifies the force transmission law.

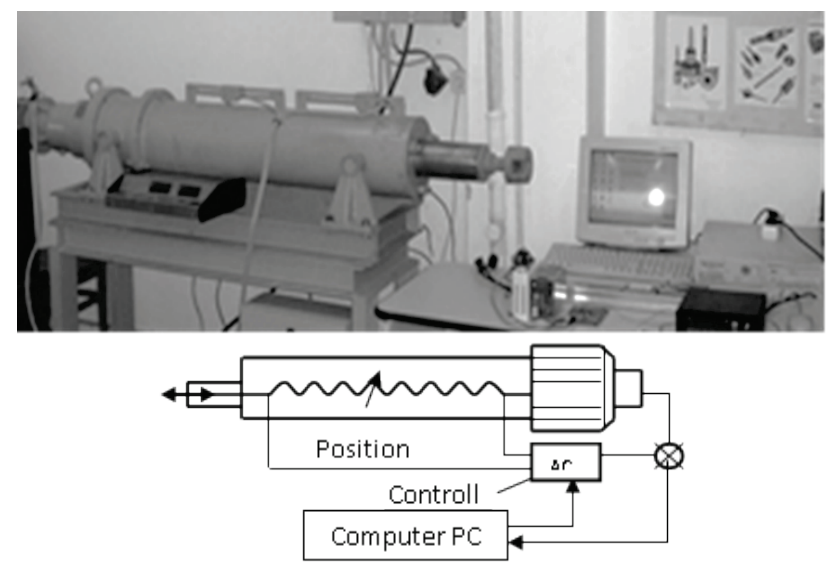

Figure 4 Rototranslation cinematic system model [16]

For the transitory running regime, the efficiency can be calculated more precisely by the correct determination of the torque loss in the two components of the reducers, including the dynamic coupling, according to the relation:

$$
\eta_{t}=\frac{M_{t}}{M_{t}+\Delta M_{c}+\Delta M_{a}}
$$

The maximum driving couple $M_{a}$ necessary to overcome inertia produced when we have accelerations is calculated based on Eq. (8). The rototranslation cinematic system model is shown in Fig. 4. [15]

$$
M_{a}=I_{A} \cdot \dot{\omega}+M_{M}+\left(K \cdot \frac{F_{0} l}{2 \pi}+\frac{P l}{2 \pi \eta}+M_{B}\right) \cdot i
$$

where: $I_{A}-$ the system inertia couple; $\dot{\omega}-$ the motor angular acceleration; $I_{a} \dot{\omega}^{2}-$ acceleration couple to overcome inertia; $M_{M}$ - internal friction couple in motor; $F_{0}$ - initial pre-compression force; $P$ - the final axial force; $M_{B}-$ friction couple in bearings; $i$ - reduction ratio.

The inertia couple for the motor driven system is:

$$
I_{A}=I_{M}+I_{R 1}+i^{2} \cdot\left[I_{R 2}+I_{S}+\frac{W}{g}\left(\frac{i}{2 \pi}\right)^{2}\right]
$$

where: $I_{M}$ - the motor couple of inertia; $I_{R 1}, I_{R 2}$ - gears' couple of inertia; $I_{S}-$ screw couple of inertia.

This measure of mechanical resistance to movement can be considered as mechanical impedance, not only from the point of view of the force, but also from the point of view of the resistant couple.

Thus, according to the definition of impedance [1]:

$Z=\frac{F}{v}$ or $Z=\frac{F}{\dot{x}}$

where: $F$ - the applied force; $v$ - the speed resulting in the point of force application.

Replacing $v=\omega r$, results:

$Z=\frac{F}{\omega \cdot r}$

where: $\omega$ - the tangential angular speed; $r$ - rotation radius.

The average coefficient of the non-uniformity of the mechanical working, by considering the movement Eq. (12) as an energy equation:

$\Delta L_{\max }=\frac{1}{2} J_{\text {red }}\left(\omega_{\max }^{2}-\omega_{\min }^{2}\right)$

where $L_{\max }$ is the excess mechanical work.

Thus the non-uniformity coefficient $(\delta)$ results, which can be calculated as follows:

$$
\delta=\frac{\Delta L_{\max }}{J_{\text {red }} \omega_{\text {med }}^{2}}
$$


where: $J_{\text {red }}-$ the inertia of motor overload; $\omega_{\max }, \omega_{\min }-$ the extreme value of the angular velocity for reduction element; $\omega_{\text {med }}-$ the average value of angular velocity.

The considerations lead us to the observation that there exists interdependence between efficiency and impedance in the dynamic regimes of modern mechanical transmissions.

When considering the inertial effect we also consider the mechanical impedance according to relation (10) with the help of the resistant couple.

\section{PROGRAMMABLE MOTION CONTROL SYSTEMS}

The block scheme of a feedback control system with actuators, which monitors position, velocity, acceleration and torque separately or like a combination, is shown in Fig. 5.
The system can be used in determination of the following kinematic characteristics of mono-axe module robots: the exact position; the law of real shift (velocitytime) of the characteristic point; the law of real shift (acceleration-time) [16].

Many motion control systems are integrated into a larger system. Thus, a more integrated motion control system would appear as shown in Fig. 5: the assembly of the process of developing new products, covering the conception aspects, manufacture and the link between them.

The application of a specific command causes a corresponding signal at the input through action input transducer. The result is an unbounded increase in controlled variable and loss of control by the command source.

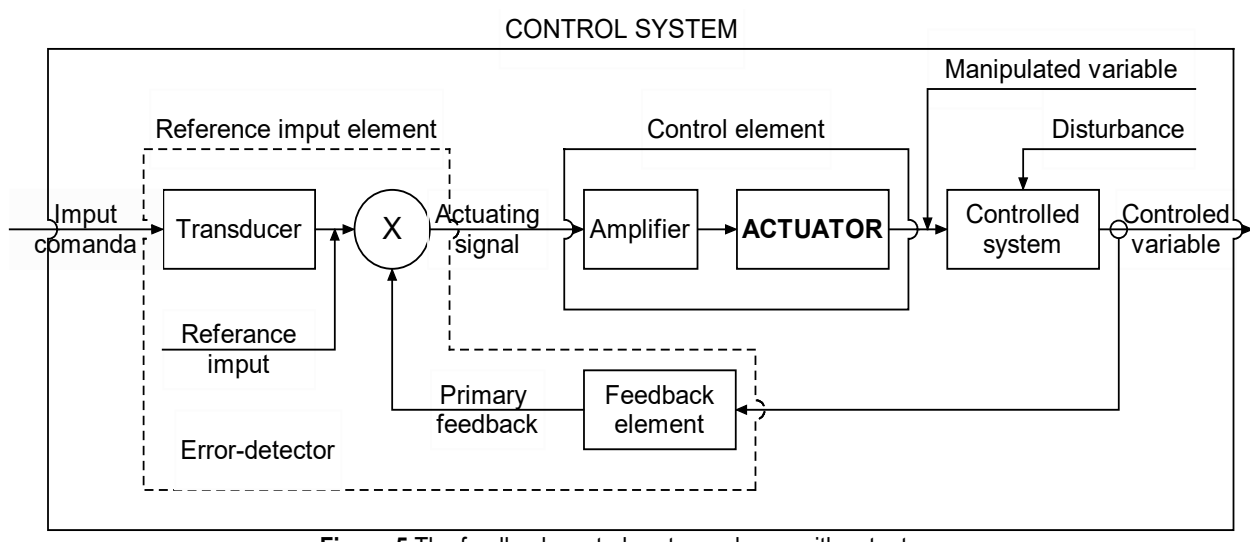

Figure 5 The feedback control system scheme with actuators

To develop an optimization pattern according to very strict engineering requirements, the introduction of several performance criteria and the formulation of some appropriate objective functions are necessary.

These restraints refer to the possibility of achieving technical performances referring to the parameters of functional geometrical precision. These are essential in the case of using robots' positioning systems, computer control machine-tools, specific to transitory conditions with frequent speeding and braking, starting and stopping at a fixed point.

The vectors to be optimized, which will satisfy the constrains, are: contact stress; basic static and dynamic axial load static axial rigidity; safety factor for outer speed; the efficiency; durability; the positional precision.

The outcome is a characteristic of functioning specific to each measure of translation unit according to the dimension and the step of the moving screw with which it is equipped.

In Fig. 6 is shown a block-diagram of the system presented in Fig. 1. We observe that the equations are the same; therefore, we can establish a correspondence among the analogous parameters which allow solving electrical circuit dynamics problems, through theoretical and experimental methods.

According to relation (1), the idealized equation for continuous control algorithm is:

$$
f(t)=k_{p} E(t)+k_{i} \int_{0}^{t} E(t) \mathrm{d} t+k_{d} \frac{\mathrm{d}}{\mathrm{d} t} E(t)
$$

where: $f(t)$ is the output at time $t ; k_{p}$ is the proportional gain constant; $k_{i}$ is the integral gain constant; $k_{d}$ is the derivate gain constant; $E(t)$ is the error at time $t$.

By adjusting the three constants of the PID controller algorithm, we can obtain the requisite process control such as response time overrun of the desired value and oscillation system. A PID controller attempts to correct the error between the measured process and set point desired.

The error correction is made by calculating and then correcting the actuator command based on three parameters. The algorithm of PID controller operation involves three separate parameters: proportional integrative derivative.

The block-diagram in the continuous control is shown in Fig. 6. This equation can be turned into a differential equation, for small sample times. The derivate term is replaced by a first-order differential equation and the integral term is approximated using trapezoidal integration. This equation required storage of the past errors.

The intermediate equation will be transformed into a recursive equation where only the previous output, current error and last two errors must be stored.

The final equation will become:

$$
F(t)=F(t-1)+k_{1} E(t)+k_{2} E(t-1)+k_{3} E(t-2)
$$


where: $F(t-1)$ - the control of previous output; $E(t-1)-$ the previous error; $E(t-2)$ - the proceeding error $E(t-1)$.

The LabVIEW program allows the selection of real numbers for gain constants.

$$
\begin{aligned}
& k_{1}=k_{p}+\frac{T k_{i}}{2}+\frac{k_{d}}{T}, \\
& k_{2}=-k_{p}-\frac{2 k_{d}}{T}+\frac{T k_{i}}{2}, \\
& k_{3}=\frac{k_{d}}{T}, T=\frac{1}{f}
\end{aligned}
$$

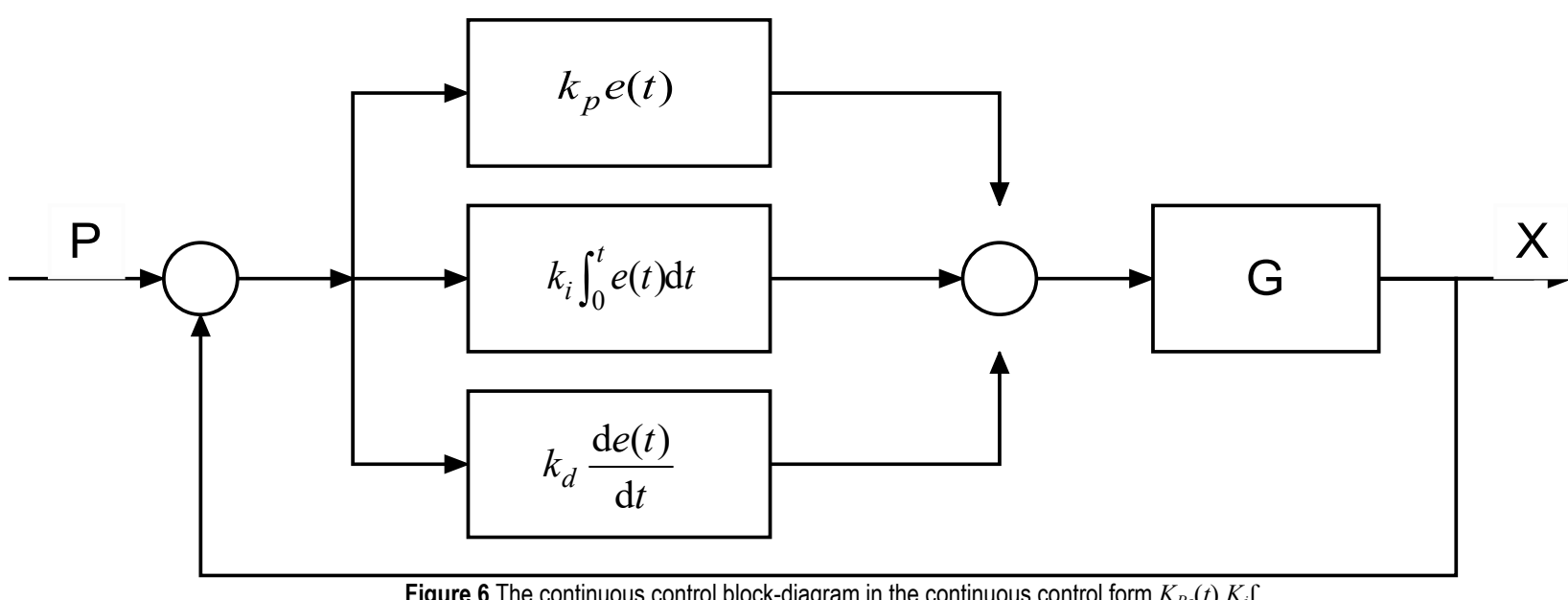

Figure 6 The continuous control block-diagram in the continuous control form $K_{P e}(t) K_{i} \delta$

\section{CONCLUSION}

The development of modern positioning servo-system type with elements of mechatronic drive technology requires additional control parameters which should verify the dynamic performance and a new concept of optimal synthesis. The results can be valued in any system of linear movement.

This paper deals with the checking and the devising of the theoretical patterns used in industry and approaching the problems to reality.

The analysis of the synthetic pattern of the actuator of the analogical servo-systems of rapid control has direct implications by deducted practical recommendations to design the transmissions of mechatronic systems. The conception and its manufacture on the computer have as application field:

- the assembly of the process of developing new products,

- covering the conception aspects, manufacture and the link between them.

The development perspectives aim to optimize the solutions, equipped with mechanisms of transmission and transformation of the movement, with high efficiency and reliability.

Developing this method on different other modular controls these researches, in future, will lead to new applications on other types of mechanical transmissions

\section{Acknowledgment}

The authors would like to thank the editorial board and anonymous reviewers for contributions made through relevant comments.

\section{REFERENCES}

[1] Mohora, C., Cotet, E., \& Patrascu, G. (2001). Simularea sistemelor de productie. Editura Academiei Romane, Bucuresti.

[2] Demian, T. s.a. (1986). The Basic of Designing the Apparatus of Fine Mechanic. EdituraTehnica, Bucuresti.

[3] Ispas, C., Predincea, N. Ghionea, A., \& Constantin, G. (1998). Maşiniunelte. Editura Tehnică, Bucureşti.

[4] Montgomery, D. C. (1996). Design of Analysis of Experiments. $4^{\text {th }}$ Edition, John Wiley \& Sons, New York.

[5] Jozwik, J. \& Czwarnowski, M. (2015). Angular Positioning Accuracy of Rotary Table and Repeatability of Five-Axis Machining Centre DMU 65 Monoblock. Advances in Science and Technology-Research Journal, 9(28), 89-95. https://doi.org/10.12913/22998624/60792

[6] Nasui, V. \& Pay, G. (2000). Basis of the Optimization of the Mechanic Efficiency. Editura Universităţii de Nord, Baia Mare.

[7] Borangiu, Th. (2003). Advanced Robot Motion Control. Editura Academiei Romane, Bucuresti.

[8] Popa, A. (2002). Controlul digital al sistemelor mecatronice. Editura Orizonturi Universitare, Timisoara.

[9] Năsui, V. (2006). Actuatoriliniari electromecanici. Editura Risoprint, Cluj Napoca.

[10] Gâlca, A. (1995). Study on the Using of Transmissions of the Field of Worm Gears in Servosystem. Editura Tehnica, Bucuresti.

[11] Hall, J. J., Williams, R. L., \& Graas, I. F. (2000). Cartesian Control for the Inertial Measurement Unit Calibration Platform. Motion \& Control No. 8, Avionics Engineering Centre, Ohio University, Athens, Ohio.

[12] Olaru, A. s.a. (1999). Dynamic robotilor industrial. Editura BREN, Bucuresti.

[13] Hvass, P. B. \& Tesar, D. (2004). Conditions based maintenance for intelligent electro mechanic actuators. The University of Texas of Austin. Austin TX 78712.

[14] Ispas, C., Predincea, N., Ghionea, A., \& Constantin, G. (1998). Mașini unelte. Editura Tehnică, București. 
[15] Nasui, V., Cotetiu, R., \& Ungureanu, N. (2012). The Multi Parametric Stand for Mechanical Testing Linear Actuator Mechanisms. Proceedings of the $3^{\text {rd }}$ International Conference on Diagnosis and Prediction in Mechanical Engineering Systems DIPRE 12, Galați.

[16] Năsui, V. \& Banică, M. V. (2014). About the Accuracy of Positioning of a Linear Robot Equipped with a High Resolution Optical System, Applied Mechanics and Materials.

\section{Contact information:}

Radu-lacob COTETIU, Prof., PhD

Faculty of Engineering, T. U. Cluj-Napoca

Dr. V. Babes street, 62A, RO-430083, Baia Mare, Romania

E-mail: radu.cotetiu@cunbm.utcluj.ro; radu.cotetiu@gmail.com

\section{Vasile NASUI, Prof., PhD}

Faculty of Engineering, T. U. Cluj-Napoca

Dr. V. Babes street, 62A, RO-430083, Baia Mare, Romania

E-mail: nasuiv@gmail.com

Adriana-Gabriela COTETIU, Prof., PhD

Faculty of Engineering, T. U. Cluj-Napoca

Dr. V. Babes street, 62A, RO-430083, Baia Mare, Romania

E-mail: acotetiu@gmail.com 